\title{
Elementos para una filosofía de las relaciones internacionales ${ }^{1}$
}

\section{Elements for a philosophy of international relations}

Recibido: 21 de mayo de 2014 - Revisado: 09 de diciembre de 2014 - Aceptado: 10 de marzo de 2015

\section{Rafat Ghotme ${ }^{2}$}

\section{Resumen}

Este artículo pretende ofrecer una alternativa al estudio del sistema internacional a través de lo que se denominará "filosofía del orden". A partir de una síntesis crítica de la teoría del caos y la teoría general de sistemas, esta propuesta analizará las dinámicas del cambio y las posibilidades que tienen los actores para determinar el curso de sus acciones. Estas acciones se circunscriben en un orden, que posibilita el cambio en su propio seno, generando las subsiguientes formas de reequilibrio del sistema y los modos de disipación del caos que los actores crean para convivir en un orden más sobrellevable. $Y$ es en este punto donde se pone a prueba la capacidad de agencia del actor internacional.

\section{Palabras clave}

Filosofía, relaciones internacionales, orden, caos.

\begin{abstract}
This article offers an alternative to the study of the international system through the so-called "philosophy of order". From a critical synthesis of chaos theory and general systems theory, this proposal will analyze the dynamics of change and the possibilities for performers to determine the course of their actions. These actions are limited within a certain order, confined in an order that facilitates the change, creating subsequent forms of rebalancing the system and the dissipation modes of chaos that actors create chaos to live together in an endurable order. And it is at this point that the agency capacity of the international actor is put to test.
\end{abstract}

\section{Keywords}

Philosophy, international relations, order, chaos.

\footnotetext{
${ }^{1}$ Este artículo es producto de una investigación adscrita a la línea estudios internacionales del Grupo de Investigación Historia Internacional, registrada en el Centro de Investigaciones de la Facultad de Relaciones Internacionales, Universidad Militar Nueva Granada, Bogotá, Colombia. 2 Candidato a doctor en Historia Política Comparada. Magíster en Historia. Licenciado en Relaciones Internacionales. Profesor asociado e investigador del Programa de Relaciones Internacionales, Universidad Militar Nueva Granada, Bogotá, Colombia. Correo electrónico: rafat.ghotme@unimilitar.edu.co Para citar este artículo: Ghotme, R. (2015). Elementos para una filosofía de las relaciones internacionales. Revista Civilizar Ciencias Sociales y Humanas, 15(28), 103-118.
} 


\section{Introducción}

En este artículo se busca vincular una filosofía de la ciencia con la disciplina de las relaciones internacionales. Tal idea surgió por dos razones fundamentales. La primera de ellas hace referencia al -peligroso- juego de tratar de predecir el comportamiento social como si se tratase del mismo mundo natural, con leyes prefijadas; a imagen y semejanza de la naturaleza, este juego supone que todo sistema social -todo orden-nace, se desarrolla y muere. Al abarcar un análisis del orden de esa manera, se asume la doble posición de incorporar supuestas leyes naturalizadas de la sociedad y, por tanto, la posibilidad de concebir que todo sistema es per se estable, ordenado.

Al estudiar la sociedad como un sistema cerrado, estable y jerarquizado, este modelo de ciencia social admite sin embargo la posibilidad del cambio: revolucionario o no, violento o aceptado, es un trastorno que se produce por circunstancias del orden mismo del sistema y, una vez dado, el sistema vuelve a su estado natural de equilibrio (Easton, 1973; Luhmann, 1993, 1998; Parsons, 1966). Las relaciones internacionales no fueron ajenas a esta concepción de una ciencia universal, un nuevo estadio de una cientificidad cada vez más integrada e interdisciplinaria (Wallerstein, 2003). De ese modo, como se verá en la siguiente sección, muchos internacionalistas de la segunda mitad del siglo XX asumieron una postura conservadora del sistema como un orden equilibrado (el orden es sinónimo de equilibrio) que se reproduce a sí mismo, como parte de su dinámica intrínseca.

La segunda razón se deriva de una supuesta reevolución de los parámetros de tal sistema social en permanente orden. Con una visión profundamente sistémica, se efectuó entre los internacionalistas un giro a partir de 1970-1980 en la concepción de la ciencia y la sociedad, adaptada desde las matemáticas y la física que implicaba la ruptura crítica entre los parámetros de la modernidad y la transición hacia la posmodernidad: nada menos que un paradigma, en el sentido que le dio Kuhn (2004), sustentado en una perspectiva caótica -no lineal ni intemporal- de la naturaleza y la sociedad.

Con la crisis de la modernidad (esto es, del racionalismo y el determinismo), una nueva filosofía de la ciencia incorporó el caos como la regla y no como la excepción en la conformación (la comprensión) de los sistemas, vistos ahora como sistemas dinámicos complejos: el caos ya no es una molesta desviación en la linealidad de los fenómenos físicos o naturales (Hayles, 1993, pp. 28-29). Aunque no era algo nuevo, la física y la matemática atrajeron a algunos internacionalistas, sobre todo por las consecuencias filosóficas que podían extraerse de su uso, esto es, las probabilidades de encuentro entre el ser y el devenir, de la liberación del ser. Al principio fueron una especie de sistémicos aislados, como Wallerstein (1991, 2004), Arrighi y Silver (2001) y AbuLughod (1989), entre otros. Al adoptar la teoría del caos asumieron que la lógica sistémica (en especial del cambio) estaba aunada a pequeñas o inapreciables fluctuaciones inmersas en un sistema en apariencia ordenado que dan paso a grandes transformaciones que modifican de manera radical la naturaleza del sistema (Abu-Lughod, 1989, p. 369). En este punto, en un momento de "bifurcación" sistémica, las probabilidades del ser de liberarse (del sistema capitalista) aumentan considerablemente.

Aunque estos teóricos asumieron un compromiso moral con la consecución de un orden justo, y que su enfoque del sistema internacional se fundamenta en un trabajo empírico muy profundo enmarcado en la sociología histórica y los sistemas de economía-mundo capitalista, se distancian entre sí a la hora de buscar posibles salidas a una era de incertidumbre. Para Wallerstein $(1984,1998,2004)$ los cambios se refieren a la transición del sistema morderno occidental a otro u otros sistemas utopísticos 
no-capitalistas, mientras que para Abu-Lughod (1989, pp. 366-368) y Arrighi y Silver (2001), podría tratarse del retorno a un sistema centrado en economías orientales, la era en la que el sistema internacional se integró por primera vez desde los parámetros del sistema de economíamundo capitalista en el siglo XIII.

En ese entonces existían diversos centros en Asia y múltiples periferias y semiperiferias en Europa, Asia y África, integradas por las rutas comerciales de los centros islámicos y China. Dejando a un lado la capacidad de acción de las organizaciones sociales antisistémicas para moldear un mundo nuevo, ¿se puede admitir que el sistema internacional volverá a presenciar esos mismos parámetros en el siglo XXI y, al mismo tiempo, un nuevo orden más justo? Un vistazo general a las economías más avanzadas de Asia, el ascenso de otras potencias no occidentales en una fase de declive hegemónico -de Estados Unidos- y el auge de la violencia terrorista, pueden apoyar este argumento, en un momento de caos sistémico.

Por otra parte, sin embargo, ¿por qué no pensar que la actual potencia en declive Estados Unidos- puede resurgir como potencia hegemónica después de una guerra colosal? Los ciclos históricos de auges y caídas de grandes imperios, ¿darán paso necesariamente a otras hegemonías imperiales, y con ello a un nuevo orden mundial? (c.f. Kennedy, 1989; Modelski, 1987).

En este trabajo, antes que responder a esas cuestiones empíricas, se pretende escudriñar -filosofar, si por filosofar entendemos algo así como deducir o pensar en lo que es- los fundamentos de un sistema que convive paralelamente en el caos y el orden $-\mathrm{o}$, para ser más precisos, sobre las posibilidades del cambio y un orden más justo. Se debe aclarar que este ensayo no pretende discutir sobre la nueva epistemología (en el sentido de una "bifurcación" en la comprensión científica de la sociedad) suscitada en las décadas de 1970-
1980; tampoco se va a realizar una heurística en torno a la teoría del caos $\mathrm{y}$, finalmente, tampoco se va a elaborar una nueva teoría del sistema internacional. Este artículo es más modesto: como todo trabajo especulativo, intenta ofrecer una alternativa al estudio del sistema internacional a través de la aplicación crítica de la teoría del caos y la teoría general de sistemas -internacional-, para llegar a proponer una alternativa intermedia en la comprensión de una era de transición caótica ${ }^{1}$.

Este enfoque confluirá en lo que aquí se denomina "filosofía del orden" en las relaciones internacionales. Tal propuesta implica una vinculación de algunos elementos provenientes de la filosofía de la ciencia con la disciplina de las relaciones internacionales. El enfoque estará puesto en las dinámicas del cambio y las posibilidades que tienen los actores para determinar el curso de sus acciones. Mediante esta "filosofía del orden", se asume que en el sistema internacional no solo están presentes los factores del orden, sino que también están dadas las posibilidades del cambio a través de los elementos del caos-desorden, generando las subsiguientes formas de reequilibrio del sistema y las formas de disipación del caos que los actores crean para convivir en un orden más sobrellevable.

"Filosofía del orden" también implica que la mayoría de los actores internacionales -así como los estudiosos internacionalistasemprenden sus acciones -o sus estudios, en el caso de los teóricos- proyectando una idea preexistente de un orden, en el que se regulan los procesos del sistema internacional. Debido a que los elementos del caos y del orden están presentes en un sistema dado, este sistema se regula a través de la figura de lo que aquí se concibe como desorden ordenado. A partir de la aplicación de la "filosofía del orden" se llegará a concluir que no todo orden dentro de un sistema conduce necesariamente a una forma más evolucionada pero estable, en la línea hacia adelante en el tiempo, sino que también es viable 
que se vuelvan a repetir algunas dinámicas acaecidas en (sub)sistemas históricos anteriores en la frontera de la modernidad ${ }^{2}$. Y es en este punto donde se pone a prueba la capacidad de agencia del actor internacional.

Para desarrollar los argumentos de la "filosofía del orden", se tomarán algunas concepciones de la sociología clásica y de la teoría del caos. Se comenzará con los fundamentos de la sociología clásica.

\section{Ciencia y orden}

Casi todos los internacionalistas prominentes de la segunda mitad del siglo XX estaban influenciados por el carácter cientifista de la sociedad, que la mostraba como un sistema cerrado, estable y jerarquizado. Estos teóricos sociales argumentaron que todo sistema social estaba regido por ciertos límites -biológicos, sociales y culturales-, en el cual los individuos se interrelacionan y reproducen los roles de acción social (Parsons, 1966) (c.f. Easton, 1973; Luhmann, 1993, 1998; Weber, 1977).

Roles, factores motivacionales y pautas de comunicación (en el sentido literal que le diera Habermas, 1998) permiten comprender la sociedad como un subproducto de la acción social. La sociedad, en ese sentido, está regida por leyes invariables e intemporales en el marco de una frontera sistémica específica. Este enfoque del sistema social, que es cerrado, no deja de admitir por esto que los sistemas no sufran cambios -literalmente no desórdenesque en últimas también forman parte de su naturaleza. El cambio, revolucionario o no, violento o aceptado, es un trastorno que se produce por circunstancias derivadas del orden mismo del sistema.

En consecuencia, todo sistema se autorregula y corre por la vía de su ordenamiento: esta visión conservadora del sistema social, que aunque acepta su dinamismo, perpetuó la idea de que todo sistema está en permanente equilibrio y orden, y al teórico, por mucho, le corresponde indagar por las condiciones en que se desequilibra -o surge el cambio, además de sus repercusiones- y vuelve a su estado natural, de orden.

Con estas premisas, la mayoría de internacionalistas de la segunda mitad del siglo XX emprendió la tarea de construir modelos de teorías de la política internacional. Como era de suponerse, la teoría abrazó hipótesis explicativas del sistema internacional en el que el orden produce el desorden y el orden. Es decir, asumieron a priori que el sistema internacional está en constante equilibrio, y que los trastornos que sufre forman parte de su dinámica intrínseca, producto de las interrelaciones de los actores o subsistemas del sistema (Kaplan, 1964; Waltz, 1988; Rosenau, 1990; Hoffmann, 1991). En otras palabras, el cambio es un agente necesario del sistema (la guerra, por ejemplo), un vacío o agujero negro que debe llenarse simplemente a través de la interacción de los actores o las instituciones internacionales. El orden, en este caso, es un medio y un fin.

No obstante, la teoría de la acción social, que concibe el sistema como uno equilibrado y dinámico al mismo tiempo, si bien admite el cambio, lo admite solo como un mecanismo inherente a la naturaleza del sistema con una orientación hacia el reequilibrio de este. El cambio entonces se piensa como una linealidad -molesta- en la que toda causa genera un efecto, sin importar sus niveles o gradaciones resultantes.

Según Parsons (1966), mediante el análisis de las repercusiones que suscita el cambio "podemos describir el estadio inicial del sistema, en que se introduce el proceso de cambio, en términos precisos y técnicos". Además, "podemos especificar lo que ha cambiado y a través de qué estadios intermedios. Si el proceso solo ha comenzado podemos especificar su dirección en relación con las varias partes del sistema" (p. 493). 
Las repercusiones pueden ser particulares o generales. Problemas como la ruptura de las "necesidades motivacionales", la estructura anárquica, las relaciones de poder, el debilitamiento del sistema de poder, las intenciones de los actores, entre otros factores, imponen tensiones que pueden introducir el cambio. Aunque existen ocasiones en que estas condiciones pueden disiparse, en otras el cambio mismo es el que se perpetúa o extiende.

Esto sucede cuando el sistema está poco integrado. Así las "tensiones solo parcialmente son dominadas, de modo que los estados crónicos de tensión llegan a estar institucionalizados y más o menos estabilizados" (Parsons, 1966, pp. 493-494). Parsons (1996) se anticipó a la teoría del caos cuando advirtió que "incluso en una sociedad relativamente estabilizada, procesos de cambio estructural están continuamente en marcha en muchos subsistemas de la sociedad, muchos de los cuales están institucionalizados" (p. 500). Sin embargo Parsons siguió fiel a su postura de un sistema naturalmente ordenado, donde el cambio -desorden o caos- es per se una condición natural del proceso dinámico de un sistema, que vuelve a ordenarse; el cambio es algo inherente a la acción social, adaptativo.

A partir de las décadas de los setenta y ochenta, por ello, algunos internacionalistas comenzaron a cuestionar la visión de un sistema per se equilibrado u ordenado. Si el sistema está en permanente estado de guerras o situaciones conflictivas; si los periodos de paz son meras ilusiones que se desvanecen en el tiempo, ¿puede tener algún sentido un término como el de sistema equilibrado y cerrado?, o mejor, ¿qué pasaría si la esencia del sistema fuera el constante desorden, caos y desequilibrio?; admitiendo como admiten los primeros sistémicos que un sistema sea afectado desde fuera, es decir, por otro u otros sistemas, ¿puede el caos tomar fuerza positiva y el derecho de producir lo mismo que el orden? (c.f. Laszlo, 1990).

\section{La teoría del caos y la ciencia social internacional: el ser y el devenir}

Katherine Hayles (1993) proporciona una definición de la teoría del caos: "En general se la puede entender como el estudio de los sistemas complejos, en el que los problemas no lineales [...] son considerados por derecho propio, y no como molestas desviaciones de la linealidad" (pp. 28-29). Un enfoque básico de la teoría del caos lo podemos encontrar en la obra de Edward Lorenz (2000), quien parte del hecho de que hay un orden oculto inmerso en los sistemas caóticos; en este sentido, el caos se concibe como un elemento claramente discernible y codificado, donde los "atractores extraños" desempeñan un rol fundamental. Los atractores extraños, en otras palabras, hacen referencia a que se puede descender ordenadamente hacia el caos, o a la inversa, sin ocuparse tanto de "las estructuras organizadas que de él emergen" (Hayles, 1993, p. 30; Lorenz, 2000).

En este trabajo, empero, se va a discutir la teoría del caos desde sus repercusiones filosóficas, antes que en los resultados prácticos ${ }^{3}$ (c.f. Arnheim, 1980; Lorenz, 2000; Gleick, 1988). Es decir, se centrará la atención en la cuestión del ser y el devenir, en la paradoja del tiempo (Prigogine, 1996; Wallerstein, 2004). Siguiendo este aspecto de la obra de Prigogine, que le apuesta al "orden a partir del caos", lo que interesa en este estudio es extrapolar las consecuencias filosóficas que devienen en ciencia (o a la inversa), sin tomar en consideración las formulaciones y los resultados de experimentos.

La teoría del caos asume que el caos es precursor del orden (y no su opuesto). Prigogine señala que el orden a partir del caos (o mejor, que un sistema no equilibrado también está ordenado) puede resolver el viejo enigma filosófico de la reconciliación del ser con el devenir, en tanto que el ser puede escoger salidas a su existencia en medio de un presente caótico en orientación a un futuro discernible, 
aunque impredecible, entre múltiples opciones. Esta reconciliación entre el devenir y el ser se haría a través de la superación de la paradoja del tiempo (Prigogine, 1996).

Para Prigogine, los sistemas son dinámicos, complejos y tienen comportamientos caóti$\cos$. Con ello se quiere decir que las ecuaciones lineales newtonianas, que marcaron la ciencia durante más de tres siglos, en donde toda causa generaba un efecto, en su secuencia, nivel y gradualidad equivalentes, en un espacio y tiempo absolutos, empezaron a tambalear: una fuerte arremetida contra la idea de la aparente intemporalidad del tiempo. A partir de ecuaciones no lineales, por tanto, se elaboró una filosofia contra el determinismo.

¿En qué radicaba la diferencia con los defensores del caos? Básicamente en una cuestión teológica: el pasado, en el primer caso, si se conocían ciertas condiciones iniciales, es la fuente providencial que desembocaría en un futuro providencial ${ }^{4}$ (Prigogine, 1996). Trasladado al historicismo, esto se traduce en que la sociedad evoluciona, mediante un encadenamiento de causas y efectos que en algún punto llegará a un fin preestablecido; en términos de ciencia social, la sociedad es representada como una especie de entidad intemporal: en los sistemas equilibrados la única historia posible es la del orden y su persistencia.

En los estudios de los sistemas dinámicos complejos, por el contrario, se entiende que todo sistema sigue un curso invariable hacia adelante en el tiempo, un tiempo creador (o destructor) que entre tantas probabilidades dadas por el azar, desordenadas e interrelacionadas (es decir, la permanente aparición de estados físicos nuevos, multiplicidad de comportamientos), se abre a nuevas probabilidades, que si bien son difíciles de predecir, dejan al ser mayores oportunidades para liberarse de su historia predeterminada. En este sentido, el tiempo es irreversible y creador.
Si bien del caos puede surgir el orden, y en todo orden pululan elementos del caos, el sistema, en últimas, se autoorganiza de una de las múltiples maneras dadas por la interrelación de los elementos en desorden y activados por una de las posibles circunstancias establecidas por el azar, siempre y cuando siga el curso hacia adelante en el tiempo. Se puede colocar el ejemplo clásico de un choque de dos autos a causa del cruce de un animal que distrajo la atención de uno de los conductores. A simple vista, el accidente estaba predeterminado o condicionado por un azar (el animal); el choque de los dos autos puede ser descrito como una interrelación de múltiples partículas o elementos que siguieron de manera inevitable hacia un curso también predeterminado por el azar.

Si se quisiera evitar ese accidente, y en general todos los hechos que nos desagradan, habría dos opciones: o mantenernos en un estado de total inmovilidad o encierro, o simplemente devolver la cinta como cuando lo hacemos con una película y en el momento del accidente apagar el video e irnos. Los sucesos acontecidos, según esta lógica, son inmodificables.

Prigogine (1996) cree que la irreversibilidad del tiempo es parte, antes que nada, de la realidad microscópica -y no de la subjetividad del observador-; la información dispersada es disipada por la interrelación del fenómeno físico, y tendría que haber una información exacta $\mathrm{y}$ una comunicación entre los elementos para devolver el tiempo asumiendo que tal información desprendiese algún elemento no disipado.

Volviendo al accidente de los dos autos, la formulación de Prigogine se entiende así: el accidente es inevitable, puesto que sigue un curso inmodificable determinado por una de las diversas opciones dejadas al azar; en segundo lugar, siguiendo a Hayles (1993) para Prigogine "el tiempo solo puede ir hacia adelante porque una barrera de información infinita divide pasado y presente" (p.130), y en este caso, incluso en el de que se pudiera devolver el tiempo, y 
el suceso se volvería a repetir, habría que interrelacionar los elementos que participaron en el hecho, pasando por todos los sucesos a través del tiempo hasta los inicios de la existencia; no solo tendría que evitarse el paso del animal por la carretera, volver al momento en que los conductores salen de sus sitios de origen, de sacar todos los conductores que estuvieron ahí en ese instante...

En el estudio del caos, pues, al tratar de resolver el viejo enigma entre el ser y el devenir, se toma una posición temporal/espacial en la que el ser admite una existencia con posibles salidas a su ignominiosa estancia en el mundo, y en la que, además, puede romper el hielo impenetrable del destino. Cuatro o cinco siglos de modernidad y racionalismo, dominados por la ciencia newtoniana, parecían estar llegando a su fin: la intemporalidad de los fenómenos, percibidos por leyes invariantes de la física, estaba dando paso a leyes impredecibles de la realidad humana -pero paradójicamente deterministas. El ser tuvo la mejor sensación de romper los tabúes que le obligaban a resignarse a su destino; ahora el ser podría escoger con algo de esfuerzo y de certeza su estancia en un mundo de incertidumbres.

\section{El caos, la historia y las relaciones internacionales.}

Enelcasodelacienciasocial, los elementos del caos-desorden, interrelacionados entre sí, con una buena dosis de azar predeterminado en el tiempo -pasado-, pueden hacer surgir nuevas formas de organización o sistemas estables. Las nuevas formas de organización que surgen de un sistema dinámico que contiene elementos del caos suscita una nueva dimensión temporal y espacial, y por tanto original. En este sentido, la teoría del caos concibe que todo sistema surge del caos.

Pero ¿es esta una relación necesaria?; ¿no puede existir la posibilidad de que tal sistema involucione? Los sistemas se vinculan con una estructura formada anteriormente. Esto lo acepta la teoría del caos, pero cabe decir un par de cosas más: o bien la irreversibilidad de los hechos forma parte de una realidad inextricable per se, o bien tales eventualidades son simplemente el resultado de subjetividades creadas por el teórico; cualquiera que sea el caso, al teórico le interesaría más la predicción y el análisis de hechos observables con orientación presente/ futuro.

Si el tiempo es una ilusión, como diría Einstein, si el pasado y el futuro son un "artilugio creado por la presencia del observador" (Hayles, 1993, p. 132), ¿cuál es el escenario que podría aportar algo para predecir comportamientos o pautas en un sistema? El meollo del asunto estriba en que el caos no acepta sin reservas la posibilidad de una ciencia determinista (Hayles, 1993); o mejor, lo que acepta de determinismo es que los sistemas siguen un curso impredecible en la línea hacia adelante en el tiempo (c.f. Wallerstein, 2004).

En una palabra: la ciencia del caos es ahistórica, de la misma manera como denunciaron la irreducible ahistoricidad de los científicos sociales de corte newtoniano. Ahistoricismo no en un sentido anacrónico, o como si los sucesos no tuviesen una estructura pasada que evolucionara hacia alguna forma determinada. Lo es en el sentido de que cuando el caos admite que los fenómenos tienen un decurso irreversible, impregnado por el azar, un solo azar predeterminado e irrepetible, per se está consintiendo también que los sucesos no volverán a ser como eran antes.

\section{La solución aportada por los teóri- cos de sistemas de economía-mundo.}

Partidarios del análisis de sistemas de economía-mundo, como Abu-Lughod (1989) o Arrighi y Silver (2001) defienden la idea de una repetición cíclica de los acontecimientos históricos producidos por los cambios sistémicos. Siguiendo los aportes de la teoría del caos, pero 
adaptada a una versión historizada de la ciencia social, aceptaron, en últimas, que la esencia del sistema internacional se descubre por medio del ordenamiento de una o más de sus múltiples posibilidades derivadas de la larga experiencia histórica.

Debido a que el sistema internacional moderno ha estado en permanentes procesos de reorganizaciones de sus fundamentos -el de la economía política global, por ejemplo-, que arrastran consigo crisis de transiciones hegemónicas, es natural entonces que el sistema mundial cambie "sustantivamente el carácter de los elementos del sistema, la forma en que estos se relacionan entre sí, y el modo en que el sistema funciona y se reproduce" (Arrighi \& Silver, 2001, pp. 28-29).

Del mismo modo, la lógica del cambio sistémico está aunada a pequeñas o inapreciables fluctuaciones inmersas en un sistema en apariencia ordenado, que dan paso a grandes transformaciones que cambian radicalmente la naturaleza del sistema (Abu-Lughod, 1989, p. 369).

De acuerdo con esto, se puede inferir que de un determinado sistema dinámico, en el momento preciso en que se modifica uno de sus parámetros -de equilibrio-, puede surgir el caos y después de ello otro orden, y así sucesivamente. A diferencia de los sistemas dinámicos ordenados, pues, que por su propia dinámica se reequilibran a partir de los cambios naturales, en los sistemas dinámicos no equilibrados, las pequeñas causas pueden generar grandes efectos $\mathrm{y}$, por tanto, cambios hacia sistemas nuevos, equilibrados o no.

La teoría del caos es válida para demostrar la no linealidad en un sistema dinámico complejo y no equilibrado. Pero su ahistoricidad ocasiona problemas. Para AbuLughod (1989) y Arrighi y Silver (2001), es probable volver a un sistema dominado por las economías (pre)capitalistas orientales, como ocurrió en el siglo XIII, pero si se sigue la lógica de la teoría del caos, esto se debería a una pura cuestión de azar. En ese sentido, la teoría del caos, cuando dice que todo sistema viaja en una línea ascendente en el tiempo, corre el riesgo de descartar posibles escenarios futuros que pueden tener semejanzas con los eventos pasados, pero, más importante aún, desconocer que se pueden repetir las condiciones estructurales de los fenónemos sociales; el riesgo radica en que la teoría del caos no acepta un grado reconocido de determinismo en la ciencia $y$, como consecuencia de ello, los sistemas más bien producen resultados drásticamente nuevos y no un simple reequilibrio del mismo sistema que lo produce. (De ese modo, la teoría del caos incurriría en una especie de eurocentrismo, o la negación de la interrelación de variables multiculturales).

Desde la perspectiva aquí propuesta, este problema se puede superar con la metodología de la investigación histórica y la filosofía de la historia: lo que se puede predecir está determinado por experiencias históricas múltiples, que en el caso de las ciencias humanas, nos puede ayudar a analizar los futuros escenarios de la sociedad en momentos de bifurcación. ¿Tan simple? Si así fuese, ya sabríamos qué tipos de sociedad, imperios, formas de pensamiento y otras estructuras surgirían en un futuro inmediato.

Pero es posible saber, sin embargo, que los subsistemas sociales -naciones o imperiosse han sucedido unos a otros inextricablemente (Duroselle, 1998). En el caso de las ciencias sociales, cuando aceptan el aporte que puede hacer la historia (algo así como leyes construidas desde la sociología histórica), esta formulación adquiere más validez: la evolución de la humanidad experimenta procesos de eterno retorno (Nietzsche, 1932). Él cree que la fuerza que obra en el universo es finita, al igual que sus variaciones, desarrollo e interrelaciones, en un tiempo infinito. Partiendo de esta premisa, en un orden se conocen fuerzas que interactúan 
en escalas de repeticiones constantes (cambio o traslaciones de los planetas, caída y emergencia de imperios, la reorganización del sistema), independientemente de los cambios acaecidos en sus formas básicas.

Debe recordarse que este es un trabajo especulativo y, por tanto, trata de mostrar cómo podría darse tal posibilidad de repetición o permanencia en un sistema histórico, admitiendo, no obstante, que se dan cambios drásticos en las estructuras del sistema. Así, lo más sugerente que se presenta en este momento del ensayo es que se va a tomar una tercera vía intermedia entre la teoría de la acción social y la teoría del caos. A partir de ello, se pretende llegar a una filosofia del orden de las relaciones internacionales.

\section{Una propuesta metodológica}

Por lo dicho hasta ahora, se puede inferir que si se intentara establecer una filosofía de las relaciones internacionales, esta se fundamentaría en una "filosofía del orden", que implica una aproximación desde la filosofía de la historia, pero, a la vez, desde una filosofía de la ciencia emancipadora. Desde esta perspectiva, la concepción de un orden en un sistema social (debe recordarse que un sistema es un concepto), aparte de estar condicionada por el conocimiento y la moral del analista, también es el producto de una experiencia histórica concreta en la que interactúan e imponen su propia idea de orden los principales actores internacionales; esto significa que los actores (y estudiosos) internacionales parten de una postura moral y/o pragmática predeterminada por la existencia de un orden, algo que finalmente los lleva a discernir la forma como se implanta, reproduce y legitima o deslegitima tal orden ${ }^{5}$.

Esto último -tanto la legitimación o deslegitimación del orden por parte de diversos actores internacionales- conlleva una distinción entre los defensores del orden y sus detractores. Desde la óptica de la teoría social, el término desorden, siguiendo a Dahrendorf (1994), es un estado en que no existe una autoridad competente reconocida por los miembros de una agrupación y en el que, por ende, no hay quien operativice las funciones administrativas. El desorden se suscita en una temporalidad bastante corta, como cuando finaliza una guerra o una revolución y hay una traslación de autoridades del antiguo al nuevo régimen.

El caos, por su parte, puede acarrear desorden: el caos se presenta como un elemento de la desviación de una linealidad -orden-que en apariencia puede ser molesta o inquietante para los sostenedores de la tradición. Además, el caos puede ser disipado o manejado por las autoridades, hasta que la acumulación de diversos momentos susciten una bifurcación perturbadora, es decir, que lo lleven al desorden total.

A lo largo de la historia del sistema internacional moderno (siglos XVI a XXI), a decir verdad, han existido pocos momentos de desorden total; han existido, más bien, múltiples momentos de caos e incertidumbre, y estos momentos son comúnmente conocidos como transiciones, como las transiciones de hegemonía de una potencia a otra decidida en una guerra general de varias décadas.

Surge de aquí una última cuestión: examinar simultáneamente la estabilidad y los cambios que se generan en un orden, partiendo de las desviaciones -lineales y no lineales- de los (sub)sistemas internacionales que se han producido en la historia moderna. En armonía con Abu-Lughod (1989), en las etapas de cambio drástico, no debería seguirse, como lo hicieran los sociólogos clásicos, con la premisa de que "las mismas causas producen los mismos efectos", un método positivista. Desde la óptica de la teoría del caos y del sistema internacional, por el contrario, la formación o reestructuración de un orden ${ }^{6}$ supone

[...] que pequeñas situaciones locales pueden interactuar con las más próximas para dar lu- 
gar a resultados que de otro modo no se habrían producido, y las grandes fluctuaciones a veces se van atenuando hasta desvanecerse mientras que otras en principio inapreciables pueden ocasionalmente irse amplificando hasta modificar la naturaleza del sistema (p. 369) (c.f. Wallerstein, 1991, p. 14).

Arrighi y Silver (2001) aseveran que en esos tipos de sistemas siempre existen ocultas ciertas regularidades caóticas (verbigracia, la tecnología, las comunicaciones, las armas, las guerras, las crisis económicas, las migraciones, los ascensos y caídas de actores estatales y no estatales, entre otras). Por tanto, el sistema tenderá a reordenarse a partir de las interrelaciones de los múltiples elementos caóticos. Cuando los sistemas se estabilizan o están ordenados (por ejemplo, con una hegemonía reconocida), el caos/desorden está presente permanentemente como pequeñas desviaciones que podrían desembocar en una bifurcación que sigue los pasos lógicos trazados por la teoría del caos, esto es, hacia un sistema de transición, y así sucesivamente (sobre los órdenes con una hegemonía reconocida (c.f. Wallerstein, 1991; 2004).

Si se acoge esta lógica, como ya se dijo, el cambio se define principalmente por los elementos del caos/des-orden, o por una cuestión de azar; ¿se debe descartar categóricamente los elementos estándares del orden? Para resolver esta cuestión, Parsons (1966) manifestaba que el análisis del cambio adquiere más importancia cuando se trata de las repercusiones antes que de las fuentes del cambio: "Las reacciones ante estas tensiones constituyen las tendencias hacia el re-equilibrio del sistema, es decir, hacia la eliminación del cambio y la restauración del estado del sistema antes de su introducción” (p. 493) ${ }^{7}$.

Esta presunción es válida, pero la teoría de la acción social no consiente el derecho propio del caos dentro del sistema, aunque algo similar se deduce de la superación de la paradoja del tiempo (como se hizo más arriba con la teoría del caos), en el sentido de que el sistema sigue un curso irreversible hacia adelante. Por ello se sugiere lograr una síntesis: los sistemas sociales pueden ser descritos como una forma de desorden ordenado, un orden que está siempre amenazado por desviaciones que producen uno o más desequilibrios (bifurcación) que desembocan en un (sub)sistema nuevo (es decir, reorganizado), y que crea sus propios mecanismos para regular el desorden o convivir con él.

Esta premisa pretende revelar una versión profundamente historizada del sistema internacional (los sucesos pueden o no volver a ocurrir tal como eran antes), mientras que su dinamismo, trayectoria y fines últimos están atravesados permanentemente por las acciones, la moral y la concepción de justicia de los actores involucrados ${ }^{8}$.

\section{Aplicación del modelo al mundo contemporáneo.}

Los (sub)sistemas internacionales también representan una forma de desorden ordenado. Aunque los diversos órdenes históricos modernos se caracterizan por tener un equilibrio natural donde acaecen los cambios como parte de su propia dinámica, cada orden internacional histórico se ha formado y ha dado paso a otros (las transiciones del subsistema westfaliano al nacionalista-popular, del nacioalista al subsistema de Naciones Unidas, de Naciones Unidas al imperialismo, del imperialismo al emancipador, etc.) gracias a la existencia de vectores que ayudan a estabilizarlo y al mismo tiempo a promover su ruptura.

Todo orden cuenta con defensores; todo orden, a su vez, tiene detractores y fuerzas destructoras. En el mundo moderno, tales fuerzas y actores son múltiples: el nacionalismo y la conformación violenta de Estados, la carrera armamentista, las guerras y las tecnologías militares creadas por Estados revisionistas, las revoluciones en las comunicaciones y los transportes, las crisis económicas cíclicas, 
las migraciones y los efectos xenófobos o la posibilidad de que las grandes olas migratorias desestructuren las sociedades de acogida ${ }^{9}$, la expansión de los actores no estatales, entre otros.

¿Cómo y cuál es el tipo de sistema internacional que se percibe en este momento? Si las relaciones internacionales "modernas" se definen como un sistema donde se yuxtaponen o superponen diversos órdenes, el actual sistema internacional no es un sistema nuevo ${ }^{10}$, sino un orden derivado de otro anterior como una secuencia de diversos momentos históricos que evolucionaron hasta convertirse en lo que es el sistema internacional contemporáneo (en otro sentido, los órdenes pueden catalogarse como sistemas o subsistemas que forman parte de una matriz general que dio inicio en la modernidad). En los límites del sistema internacional anárquico, dominado por una o más potencias, estas generan normas de comportamiento comúnmente aceptadas durante algún tiempo (esto es, los más interesados en mantener el orden), y en paralelo conviven con elementos del caos (desde el punto de vista de los actores relevantes o dominantes) que pueden llevar al cambio. En el sistema internacional pueden existir varios órdenes:

- Hegemónico: una gran potencia domina en el sistema e intenta legitimarse como la abanderada en la tarea de la disipación del caos y en últimas del desorden. La potencia hegemónica busca eliminar o subordinar a los competidores (que según su entender son los portadores del caos). Asimismo crea un orden a través de normas, presentándolas a los demás como normas benevolentes (Mearsheimer, 2001; Walt, 2005).

- Equilibrio: varias potencias dominan en el sistema, intentando legitimarse como los valedores del orden y asegurando a sus satélites la supervivencia. La diplomacia y el derecho internacional buscan disipar el caos y el desorden (Aron, 1963; Waltz, 2000).
- Acomodamiento: es una fase intermedia entre la hegemonía y el equilibrio. Los Estados medios procuran obtener beneficios de la potencia hegemónica, tales como seguridad y un orden económico liberal y abierto, mientras no se amenace su soberanía política. Sin embargo, solo se acomodan mientras logren equilibrarse (Walt, 1987).

- Disidencia: existen múltiples tipos de actores estatales y no estatales que desafían el orden hegemónico o en general a los defensores del sistema dominante (capitalista-estadocéntrico). Los defensores del orden vigente representan a esos actores de múltiples maneras: Estados canallas, terroristas, etc. A la inversa, estos actores acusan a los reproductores del orden de representar el mal y la injusticia.

- Transición: los elementos del caos y el eventual desorden sistémico adquieren mayor notoriedad, apuntalando las acciones de los actores insatisfechos con el orden. Existen todo tipo de actores que reivindican una posición más favorable, desde los Estados revisionistas hasta los actores no estatales que usan la violencia. Por último, se da paso a otro orden ${ }^{11}$.

El sistema internacional contemporáneo se caracteriza por estar en una fase de transición hegemónica. En ese sentido, sobresalen elementos del caos o, alternativamente, un conjunto de actores que ha decidido no admitir más el tipo de orden impuesto: el orden promueve un ideal, una muestra de la posibilidad de liberación que sigue determinando el comportamiento en sociedad.

\section{Recapitulación}

En este artículo se pretendió mostrar que las relaciones internacionales pueden abordarse 
a partir de una concepción de orden: un orden establecido, que contiene elementos del caos y que trata de regularlo.

Los órdenes, en este sentido, cuentan con algunos "vectores" caóticos conectados entre sí. A diferencia de la ciencia social clásica, tales vectores no son una simple desviación de la linealidad presente en una sociedad o sistema social particular, sino parte inherente de este y que promueven el cambio sistémico por derecho propio.

La filosofía de las relaciones internacionales propuesta aquí enmarca el objeto formal de estudio de las relaciones internacionales en un sistema que por su propia naturaleza es ordenado, pero que consta de elementos caóticos que lo dinamizan: el sistema está en un permanente estado de desorden ordenado, donde los actores relevantes del sistema tratan de convivir con el caos creando mecanismos de regulación o tratando de disiparlo. A la inversa, los vectores del caos -actores que usan diversos mecanismos- dirigen sus esfuerzos para cambiar el orden.

A esta presunción se le denominó "filosofía del orden". Para ello, se tomó como punto de partida las premisas de la sociología clásica expuesta por Parsons y las críticas aportadas por la teoría del caos. Sometida a un escrutinio filosófico, se buscó mostrar que sus postulados siguen siendo útiles, y que es útil lograr una síntesis. La teoría del caos, en ese sentido, se sometió a un balance crítico para sugerir que sus contribuciones son válidas para demostrar la no linealidad y la importancia del caos en un sistema dinámico complejo, no equilibrado; pero también se mostró que en cierto modo es una propuesta ahistórica, a pesar de reconocer que todo sistema produce uno nuevo y asegura una continuidad parcial de la antigua estructura del sistema. En términos generales, la teoría del caos acepta que todo sistema sigue una línea hacia adelante en el tiempo, sin admitir, por tanto, las posibilidades de repetición o continuidades cíclicas de los sistemas sociales.

De ello se deriva otra importante conclusión: el sistema internacional presupone un estudio hondamente historizado. En el estudio de la sociedad es más legítimo centrarse en el descubrimiento de probabilidades en el futuro que en leyes universales. Desde el punto de vista metodológico, el analista realiza sus estudios partiendo de un cúmulo de hipótesis explicativas que presuponen una lógica sistémica de los fenómenos internacionales acaecidos a lo largo de la historia. Los acontecimientos o fenómenos del sistema internacional son construcciones históricas que fluctúan en orientación presente/futuro.

En este sentido, las relaciones internacionales, como disciplina científica, pueden asemejarse a algo así como una historia del presente del sistema internacional: un sistema es una construcción histórica en la que puede determinarse la existencia de un orden, con sus dinámicas de caos y reordenamiento, que bien pueden ser producto de factores inherentes al sistema, o bien pueden estar condicionadas por posturas morales del analista, que crea un marco de referencia de acuerdo con el espíritu reinante de su entorno, cultura o civilización.

Dicho en otros términos: un orden se construye históricamente tomando como referente la pretensión de la realización humana sustentada en el pasado; un orden, además de ser la figuración -o configuración- de la narración histórica, de los filósofos o pensadores de una época determinada, también es el resultado de una sociedad real con personas de carne y hueso que son (o deberían ser) conscientes de su condición de "seres históricos".

Por lo demás, es posible que se suscite en su propio seno las contradicciones que llevarán a algunos actores del orden a promover la existencia de un orden nuevo. Aunque el orden y el caos puedan ser condicionantes estructurales, 
el hecho es que real o empiricamente el analista emprende sus estudios aceptando la existencia apriorística de un orden, y su propósito último se centra en descifrar los "vectores" que lo desordenan y las instituciones que se crean para regularlo -y en este caso específico para sostener o trastornar el orden-.

El orden, de otra manera, es la proyección de una realidad histórica. Pero debido precisamente a que las relaciones internacionales dejan abierta la posibilidad del cambio (¿acaso el ser humano no es creador de ideas, no es capaz de modificar el curso de la historia?), surge entonces la tarea difícil y larga de juzgar la factibilidad de un proyecto de orden internacional justo. Queda por resolver si tal proyecto es posible gracias a la acción humana y sus juicios de valor, o si tales acciones quedarán sujetas en exclusiva a la bruma de la historia y a la indiferencia de un amplio sector de la humanidad.

\section{Notas}

${ }^{1}$ Aunque los elementos que aporta la constatación empírico-histórica son indispensables, en este trabajo van a prevalecer los elementos de juicio deductivo-inferenciales. Esto, sin embargo, ha sido abordado en otra parte (Ghotme, 2011).

${ }^{2}$ En casi todos los casos, si no en todos, esto último termina desempeñando un rol praxeológico y moral en torno a las posibilidades de un orden más justo: el analista, incluso el más acendrado de los cientifistas, le incorpora su propio compromiso moral a la disciplina. Aquí no nos ocuparemos de esto.

${ }^{3}$ Desde el punto de vista físico y matemático, las auto-organizaciones o estructuras disipativas provienen de sistemas fuera de equilibrio que surgen del caos, sobre todo cuando la producción de entropía es alta, y no al contrario, como creyeron algunos sociólogos como Parsons (1966, p. 498), y a diferencia de Lorenz (2000), quien considera que la complejidad de la naturaleza permite reconciliar las simetrías y asimetrías para suministrar nueva información: su dinámica no lineal muestra que está llena de sorpresas y desorden (lo que importa es la información y su renovación a través del caos).

${ }^{4}$ Tal era la versión de Parsons (1966, pp. 498-499) sobre la direccionalidad y la entropía: "En física no es en absoluto imposible que la entropía de un sistema se reduzca en lugar de aumentar". No "puede mantenerse una concepción lineal general de la evolución de los sistemas sociales. El proceso, considerado en uno de esos sistemas, no puede ser simplemente un cambio al azar desde un estado del sistema a otro". Como se observa, esta versión clásica de la sociología, categóricamente -casi siempre injustamente- fue atacada por los teóricos del caos, sobre todo cuando tratan de responder sobre la linealidad de algún fenómeno físico (sobre la entropía y la percepción del ser, el orden y el desorden, c.f. Arnheim, 1980).

${ }^{5}$ Sin entrar en el debate, se puede remitir a la obra de Gleick (1988), para quien existe un punto intermedio donde la teoría general del caos presenta al caos en sistemas complejos donde puede convivir, a la vez, con los atractores extraños y el orden a partir del caos (c.f. Hayles, 1993).

${ }^{6}$ Para los científicos sociales positivistas no importa establecer causas primeras o propósitos últimos, sino el estudio de hechos observables o "reales" con una fuerte predisposición a la búsqueda de leyes o generalizaciones.

${ }^{7}$ En caso de que pudiera determinarse una "filosofía del orden", esta se identificaría con la idea reguladora del saber totalizado. Desde Kant o Fichte, la weltanschauung de los seres del filósofo, más bien- concibió la existencia de un mundo, su entorno, historia y la diferencia con otros, como un orden determinado por una idea. Sin embargo, la existencia de un cúmulo de valores en un orden dado se suscita precisamente en una premisa, la de la historia concreta 
(c.f. Sartre, 1963). Las posibilidades de existencia, la trascendencia o el ideal de un orden solo podrían ser perceptibles en la medida que -también por medio de la historia- se conjuguen un sinfín de acontecimientos, como las revoluciones o la guerra. El estudio del orden es el que nos hace captar una realidad social que imbrica una evolución de forma sistemática, que se relaciona con un entramado de procesos y lógicas propios de la historia. Una crítica de la razón dialéctica, como entendemos la de Sartre, empero, le da prelación a una concepción del orden como la realización de las experiencias humanas concretas; su idea de un saber totalizado o idea de un mundo moderno entra en la relación dialéctica con el mundo de la experiencia, entonces, más o menos supeditada a este último (c.f. Duroselle, 1998; Schwarzenberger, 1960).

${ }^{8}$ Dentro de estos componentes sobresalen al menos dos: a) las crisis de transiciones hegemónicas en el sistema mundial, incluyendo las civilizaciones no occidentales; y b) la reconfiguración parcial o total del capital en función del Estado moderno, la empresa y los trabajadores (Arrighi \& Silver, 2001; Wallerstein, 1996).

9 Talcott Parsons (1966) creía que "una teoría general de los procesos de cambio de los sistemas sociales no es posible en el presente estadio de la ciencia. La razón de ello es muy simple: semejante teoría implicaría un conocimiento completo de las leyes del proceso del sistema y este conocimiento no lo tenemos" (pp. 484-485), aunque fuera posible tener un conocimiento de los subprocesos particulares de cambio dentro de esos sistemas.

${ }^{10}$ En todo sistema subyacen elementos (exógenos o endógenos a él) que determinan su existencia, desarrollo y dinamismo. Tales elementos suelen ser perdurables en el tiempo, y en la medida que se modifican (caos-resquebrajamiento-transición), también se mantienen elementos "viejos" (regularidades) que o bien se transforman (pero mantienen su esencia) o bien perduran. Desde ese punto de vista, enton- ces, cabe la posibilidad de que se repitan una o más de las diversas posibilidades que existieron en el pasado.

${ }^{11}$ Este es el caso de las migraciones centroamericanas y mexicanas a Estados Unidos, y de los africanos y europeos orientales a Europa occidental (c.f. Rosenblum, Kandel, Seelke, \& Wasem, 2012).

${ }^{12}$ Lo que cambia realmente no es el sistema, cambia son los órdenes o subsistemas: esta premisa viene de la teoría neorrealista según la cual el cambio se presenta en la estructura de poder, el paso de un orden unipolar a bipolar o multipolar, o a la inversa. Un cambio de sistema como tal, se daría cuando los elementos de la era moderna (anarquía, estatismo, capital) den paso a otro mundo, no dominado por los Estados y el capitalismo.

${ }^{13}$ Como se puede notar, se plantea aquí una versión del orden eminentemente "realista". Estos aspectos se desarrollan de manera empírica en Ghotme (2006, 2011).

\section{Referencias}

Abu-Lughod, J. (1989). Before European hegemony. The World System A.D. 1250-1350. Nueva York: Oxford University Press.

Arnheim, R. (1980). Hacia una psicología del arte. Arte y entropía (Ensayo sobre el desorden y el orden). Madrid: Alianza Editorial.

Aron, R. (1963). Paz y Guerra entre las Naciones. Madrid: Alianza.

Arrighi, G., \& Silver, B. (2001). Caos y orden en el sistema-mundo moderno. Madrid: Akal.

Daherendorf, R. (1994). Ley y orden. Madrid: Civitas Ediciones. 
Duroselle, J. B. (1998). Todo imperio perecerá. Teoría sobre las relaciones internacionales. México: Fondo de Cultura Económica.

Easton, D. (1973). Enfoques sobre teoría politi$c a$, Buenos Aires: Amorrortu.

Ghotme, R. (2006). Orden, des-Orden y antiorden mundial. Problemas de Relaciones Internacionales. Revista de Relaciones Internacionales, Estrategia y Seguridad, 1(2), 75-103.

Ghotme, R. (2011). La configuración del poder en el sistema internacional contemporáneo. Revista de Relaciones Internacionales, Estrategia y Seguridad, 6(1), 74-201.

Gleick, J. (1988). Caos. La creación de una ciencia. Barcelona: Seix Barral.

Habermas, J. (1998). Facticidad y validez. Madrid: Trotta.

Hayles, K. (1993). La evolución del caos. El orden dentro del desorden en las ciencias contemporáneas. Barcelona: Gedisa Editorial.

Hoffmann, S. (1991). Jano y Minerva: ensayos sobre la guerra y la paz. Buenos Aires: Grupo Editor Latinoamericano.

Kaplan, M. (1964). System and process in international politics. Nueva York: Science Editions.

Kennedy, P. (1989). Auge y caída de las grandes potencias. Barcelona: Plaza y Janés Editores.

Kuhn, T. (2004). La estructura de las revoluciones cientificas. México: Fondo de Cultura Económica.

Laszlo, E. (1990). La gran bifurcación. Crisis y oportunidad: anticipación del nuevo pa- radigma que está tomando forma. Barcelona: Gedisa.

Lorenz, E. (2000). La esencia del caos. Un campo de conocimiento que se ha convertido en parte importante del mundo que nos rodea. Madrid: Debate.

Luhmann, N. (1993). Teoría Politica en el Estado de Bienestar. Madrid: Alianza Editorial.

Luhmann, N. (1998). Sistemas sociales: lineamientos para una teoría general. Barcelona: Anthropos.

Mearsheimer, J. (2001). The Tragedy of Great Power Politics. Nueva York: Norton.

Modelski, G. (1987). Long Cycles in World Politics. Londres: Macmillan.

Nietzsche, F. (1932). El eterno retorno. Obras Completas (Tomo IV). Madrid: M. Aguilar Editor.

Parsons, T. (1966). El Sistema Social. Madrid: Alianza.

Prigogine, I. (1996). El fin de las certidumbres. Santiado de Chile: Editorial Andrés Bello.

Rosenau, J. (1990). Turbulence in World politics: a theory of change and continuity. Princeton: Princeton Press.

Rosenblum, M., Kandel, W., Seelke, C., \& Wasem, R. (2012). Mexican Migration to the United States: Policy and Trends. Washington D.C: Congressional Research Service.

Sartre, J. P. (1963). Teoría de los conjuntos prácticos. Critica de la razón dialéctica (Tomo I). Buenos Aires: Editorial Losada.

Schwarzenberger, G. (1960). La política del poder. Estudio de la Sociedad Internacional. México: Fondo de Cultura Económica. 
Wallerstein, I. (1984). El moderno sistema mundial. El mercantilismo y la consolidación de la economía mundo europea, 1600-1750. México: Siglo XXI editores.

Wallerstein, I. (1991). Geopolitcs and Geoculture. Essays on the changing world-system. Cambridge: Cambridge University Press.

Wallerstein, I. (1996). Después del liberalismo. México: Siglo XXI editores.

Wallerstein, I. (1998). Utopística o las opciones históricas del siglo XXI. México: Siglo XXI editores.

Wallerstein, I. (2003). Abrir las ciencias sociales. México: Siglo XXI Editores.
Wallerstein, I. (2004). Capitalismo histórico y movimientos antisistémicos. Un análisis de sistemas-mundo. Madrid: Akal.

Walt, S. (1987). The origins of alliances. Ithaca: Cornell University Press.

Walt, S. (2005). Taming American Power: The Global Response to U.S. Primacy. Nueva York: W.W. Norton.

Waltz, K. (1988). Teoría de la Política Internacional. Buenos Aires: Grupo Editorial Latinoamericano.

Waltz, K. (2000). Structural Realism after de Cold War. International Security, 25(1), 5-41.

Weber, M. (1977). Estructuras de poder. Buenos Aires: Editorial La Pléyade. 